\section{Improvement of European translational cancer research. Collaboration between comprehensive cancer centers}

\author{
Ulrik Ringborg ${ }^{1}$, Dominique de Valeriola ${ }^{2}$, Wim van Harten ${ }^{3}$, \\ Antonio Llombart Bosch ${ }^{4}$, Claudio Lombardo5, Kenneth Nilsson ${ }^{6}$, \\ Thierry Philip ${ }^{7}$, Marco A Pierotti ${ }^{8}$, Peter Riegman ${ }^{9}$, Mahasti Saghatchian $^{10}$, \\ Guy Storme ${ }^{11}$, Thomas Tursz ${ }^{10}$, and Dirk Verellen ${ }^{11}$ \\ ${ }^{1}$ Cancer Center Karolinska, Karolinska University Hospital, Sweden; ${ }^{2}$ Institut Jules Bordet, Belgium, \\ ${ }^{3}$ The Netherlands Cancer Institute, Netherlands; ${ }^{4}$ Fundacion Instituto Valenciano de Oncolgia, Spain; \\ ${ }^{5}$ Istituto Nazionale per la Ricerca sul Cancro, Italy; ${ }^{6}$ Department of Pathology, University of Uppsala, \\ Sweden; ' Leon Berard, France; ${ }^{8}$ Fondazione IRCCS Istituto dei Tumori, Italy; ${ }^{7}$ Erasmus Medical Center, \\ Netherlands; ${ }^{10}$ Institut Gustave-Roussy, France; ${ }^{11}$ Universitair Ziekenhuis Brussel, Belgium
}

\section{ABSTRACT}

Even though the increasing incidence of cancer is mainly a consequence of a population with a longer life span, part of this augmentation is related to the increasing prevalence of patients living with a chronic cancer disease. To fight the problem, improved preventive strategies are mandatory in combination with an innovative health care provision that is driven by research. To overcome the weakness of translational research the OECI is proposing a practical approach as part of a strategy foreseen by the EUROCAN+PLUS feasibility study, which was launched by the EC in order to identify mechanisms for the coordination of cancer research in Europe.

\section{European cancer research}

Cancer is becoming one of the most important chronic diseases and, at the same time, in a global perspective, according to calculations by the World Health Organization, is causing more deaths than AIDS, malaria and tuberculosis together ${ }^{1}$.

European cancer research has been thoroughly analyzed in a project financed by the European Commission after a request of the European Parliament. This project involved a large number of European cancer centers and other stakeholders and was reported to the European Commission in early 2008. European cancer research has often been criticized for being fragmented with insufficient collaboration between research key players. From the political perspective it seems that research has not been sufficiently coordinated with a focus on improving survival and quality of life for cancer patients. In other words, there seems to be a gap between the potential to innovate cancer care and the actual situation in the provision of oncologic care.

The project reports a number of interesting strengths in European cancer research, especially in the global perspective of basic and preclinical cancer research. There are health care organizations in several countries performing populationbased studies. Some countries have well-developed patient databases. The potential to establish high quality bio-banks is another advantage especially when electronically connected to databases with clinical information related to the patient involved. A number of technical platforms supporting clinical research are available. Collaborative networks for cancer clinical trials, among which the EORTC (European Organization for Research and Treatment of Cancer) is the most comprehensive one, make Europe an interesting area for the expansion of clinical trials driven by biological questions. Furthermore, the intellectual capital is impressive but vulnerable, since it is of interest for research centers outside Europe, often of-
Key words: cancer, translational research, comprehensiveness, coordination.

Correspondence to: Ulrik Ringborg, Cancer Center Karolinska, Radiumhemmet, Karolinska University Hospital Solna, S-171 76 Stockholm Sweden.

Tel 46-8-517 72121; e-mail ulrik.ringborg@karolinska.se 
fering conditions that could attract researchers with high potential.

Nevertheless, the report points at a number of weaknesses, most of which are already known by researchers. The research process from basic to clinical research is often fragmented with the consequence that the time from basic discoveries to clinical implementations of new diagnostic and treatment methods is too long. The rapid expansion of new information in cancer biology together with insufficient communication between basic and clinical researchers create a gap between basic and clinical research. Both funding and education are contributing to maintain this gap. Clinical research involving patients is often more time consuming especially in relation to the formal aspects and the administrative burden is therefore a bottle neck in the research process. Another gap is identified in between clinical research and the implementation of new technologies into cancer care. The increasing complexity of research causes problems in reaching the critical mass for several types of cancer research leading to unnecessary delays in publishing studies based on adequate series. An important contributing factor is combining excellent research with the needs of patients. Cancer care of high quality is impossible without a multidisciplinary approach integrating various treatment modalities and diagnostic functions. Research adapted to these needs must be oriented towards this multidisciplinary approach for effective patient-focused research. Tumor diseases, although originating in different organs, have common biological traits such as the dissemination process. Research on the mechanisms of metastasis formation and its prevention should be the main focus of cancer research. Within this background, one of the important conclusions from the Eurocan+Plus project is that present fragmentation can be overcome through a more rational integration of the cancer research process, translational research, and an increased collaboration between centers in order to reach the critical mass for most types of cancer research.

\section{The Comprehensive Cancer Center}

The main goals of the activities to combat cancer are to decrease mortality and morbidity and increase survival and quality of life. To reach these goals we need care and prevention which are integrated with research and education. A type of organization where this integration is achieved is usually called a comprehensive cancer centre (CCC).

A CCC provides patients with care of high quality with a focus on the needs of patients.

To cover the needs in the total clinical pathway a number of competences are needed: diagnostic activities represented by pathology/cytology, imaging and laboratory medicine, individual molecular diagnosis; surgical specialities, radiation therapy and medical oncology; psychosocial oncology, rehabilitation, supportive care and palliative oncology. In a CCC individual focused prevention and early detection should be offered to potential patients.

Research in a CCC should be translational cancer research, which may be defined as a research continuum from basic through preclinical and clinical research and implementation and evaluation in the routine care. Or, as stated by the NCI Translational Research Working Group, translational research transforms scientific discoveries arising from laboratory, clinical or population studies into clinical applications to reduce cancer incidence, morbidity, and mortality. The presence of expertise in epidemiology, bio-statistics, bio-informatics in a CCC should be then considered an added value.

Education in a CCC aims at all functions in the centre. Basic education of medical students and nurses as well as specialist training and continuous medical education are missions of the CCC. Research education plays a crucial role, which is an absolute priority if the complex research process is maintained. The CCC should also have the role to contribute to educational efforts to improve prevention. Another important function is to supply decision makers with information on problems and strategies to improve cancer care.

A CCC may be a cancer hospital with sufficiently developed structures for research and education. This is the case for the most prominent American CCCs and in some European countries CCCs of this type have been established. However, in Europe cancer care and research are often part of a university hospital. In this case the organization is often fragmented and should be improved to form a more visible and identifiable CCC. An advantage, however, is the close geographic relationship to other disciplines of interest for cancer patients often suffering from other diseases than cancer. For both forms of organization there are often hospitals in the neighborhood taking care of cancer patients. The OECI's view is to create the functional network around a CCC to assure the quality of cancer care and to stimulate research collaboration thereby extending the comprehensiveness outside the centers. It is important to emphasize the importance of a strong core containing highly specialized health care, advanced research resources and structures for education in a comprehensive cancer network.

\section{OECI}

The OECI is a non-profit organization and a European Economic Interest Grouping, thus having a legal status which is visible within the European Union. It is the only organization built on cancer centers/institutes as members linking 58 centers at present. The OECI main mission is to stimulate the development of CCCs 
in Europe and facilitate collaboration between such centers. According to the OECI mission statement ${ }^{2}$ conventional project collaboration between research groups is not enough. The increasing complexity in both the multidisciplinary cancer care and translational research requires a new type of closer collaboration between centers to put the most important research questions from the patients perspective in focus. Increased research collaboration is necessary for studies not only of patients with rare tumors but also of subgroups of other patient groups. The expensive infrastructures needed for modern translational cancer research may be difficult to iterate in all centers. Therefore we need to share and harmonize structures, for instance clinical trials units for biologically driven clinical trials, bio-banks, platforms for biomics, patient data registries. The need for competences is increasing and should be shared to a larger extent to include educational programs.

The OECI has a number of working groups covering activities supporting the development of comprehensiveness. An accreditation methodology for European CCCs is the mission of one of the groups. A questionnaire regarding quantitative characteristics of cancer centers, a quality assessment manual and a procedure for auditing centers will also be used and are presently part of a pilot study of a limited number of centers. The methodology will be available for all OECI members in fall this year. Parallelly, the first step toward a labialization/designation system will be taken to define the OECI criteria for a European CCC. The next step will be to develop criteria linked to quality measurements to identify CCCs of excellence.

A bio-pathology working group is linked to the virtual European bio-bank, TuBaFrost, which is under the umbrella of the OECI. Work is ongoing to implement TuBaFrost in the OECI centers. An educational working group supports the development of comprehensiveness. A working group for new technologies supports members to improve the infrastructures for radiation therapy and imaging. For development of clinical guidelines the OECI is collaborating with the EU project CoCanCPG.

The OECI is a growing organization. The need to create closer collaboration between centers is increasing. No center has the critical mass necessary for most types of cancer research. Multidisciplinarity is fundamental for improving cancer care. The research needed for the development of multidisciplinary care is complex and this complexity necessitates the collaboration between CCCs.

\section{A network of centers. A solution to reach the critical mass for most types of cancer research}

Modern translational research requires new types of infrastructures, linkage of competences to cover differ- ent parts of the research process, patients and biological materials from increasing subgroups of cancer patients but also from patients with rare tumor diseases. For therapy development new molecular targets and new treatments are needed. To optimize treatments personalized cancer medicine should be an important focus for research. Fundamental will be the identification of biomarkers for patient and disease characteristics as well as the prediction of response to treatments. Such a development must be seen in the context of a multidisciplinary care approach of the patient. The potential to innovate and improve cancer treatment is spectacular if modern cancer biology, genetic engineering, methodological developments to use biological materials from patients and clinical questions are analyzed in a holistic way. Some of the entities organizing European cancer centers work, each from a different perspective, towards establishing a coherent and advanced level of cancer research.

The OECI has had the objective to stimulate collaboration between centers for a long time. If such collaboration is effective, centers should provide the researchers with infrastructures for translational cancer research which should be harmonized between centers. A similar strategy has now been developed and is under implementation within the EORTC (European Organization of Research and Treatment of Cancer) - NOCI (Network Of Core Institutions). This network aims at a formal collaboration between a limited number of centers with a substantial number of patients and strong infrastructures for translational research to conduct biologically driven clinical trials.

An important conclusion of the Eurocan+Plus project was to propose the formation of a European translational research platform by linking the most research oriented CCCs and basic/preclinical cancer research centers. Such platform should provide Europe with a cancer research infrastructure where the European research strengths can be used in a rational way. On November 6, 2007, representatives of 19 European centers met in Stockholm to discuss this concept and the discussion resulted in the "Stockholm Declaration"3. A number of objectives of the platform were identified: to define and coordinate specific areas for research collaboration; to harmonize and share infrastructures and competences; to optimize information exchange between centers; to improve mobility of researchers within the platform; to develop research education in order to attract young investigators and retain them in Europe; and to speak for translational cancer research with one voice in Europe.

A platform of this type must be dynamic. It may start small but have the potential to grow. The platform shall be open so new centers can join in future, and through the dissemination of information the platform will be of value also for centers outside the platform. A well organized translational cancer research platform should 
represent a "world-class infrastructure" which is proposed in the Green Paper, a strategic document from the European Commission. It is interesting that the majority of the centers behind the Stockholm Declaration are members of the OECI. Further, it was decided that the OECI accreditation methodology will be used to identify criteria for an objective selection of centers for the platform.

\section{References}

1. Ferley J, Bray F, Pisani P, Parkin DM: IARC CancerBase No5 version 20. IARC Press, Lyon, France. GLOBOCAN 2002: Cancer Incidence, Mortality and Prevalence Worldwide.

2. Ringborg U, Pierotti AM, Storme G, Tursz T, A European Economic Interest Grouping. Managing cancer in the EU: The Organisation of European Cancer Institutes (OECI). EJC in press.

3. Ringborg U: The Stockholm Declaration. Molecular Oncology, 2008, doi:10.1016/j.molonc.2008.03.004. In press. 